\title{
Two-stage total hip arthroplasty for primary advanced septic arthritis of the hip in adults: comparison of debridement versus femoral head resection with spacer implantation
}

\section{Zhenzhong Li}

The Third Hospital of Hebei Medical University

\section{Congcong Wei}

The Third Hospital of Hebei Medical University

\section{Xiangke Li}

The Third Hospital of Hebei Medical University

\section{Mengxuan Yao}

The Third Hospital of Hebei Medical Hospital

Huijie Li ( $\square$ lihuijiework1@sina.com )

The Third Hospital of Hebei Medical University

\section{Research article}

Keywords: Primary septic arthritis, Debridement, Antibiotic spacer, Two-stage total hip arthroplasty

Posted Date: January 21st, 2020

DOI: https://doi.org/10.21203/rs.2.21439/v1

License: (c) (i) This work is licensed under a Creative Commons Attribution 4.0 International License.

Read Full License 


\section{Abstract}

\section{Background}

To compare the outcomes of debridement and antibiotic-loaded spacer implantation in primary advanced septic arthritis (SA) of the hip in adults treated with two-stage total hip arthroplasty (THA).

\section{Methods}

In the first stage, 9 patients were treated with debridement and 11 patients were treated with antibioticloaded spacer implantation. Then, THA was conducted in all patients in the second stage. Patients were evaluated based on the recurrence of infection, Harris hip score, visual analogue scale (VAS) pain score and leg-length discrepancy.

Results

No cases of infection, deep vein thrombosis, death, and loosening of hip prosthesis were observed during follow-up. The mean follow-up time was $29.09 \pm 10.80$ months in the debridement with THA group, and $28.22 \pm 14.80$ months in the spacer implantation with THA group. Before the THA surgery, the mean leglength discrepancy was $2.80 \pm 2.03 \mathrm{~cm}$ in the debridement with THA group, and $0.50 \pm 0.23 \mathrm{~cm}$ in the spacer implantation with THA group $(P<0.05)$. In the latest follow-up, The Harris hip scores of patients was $90.33 \pm 4.85$ in the debridement with THA group and $94.36 \pm 2.34$ in the spacer implantation with THA group $(P<0.05)$, respectively. There was no statistical significant difference in VAS pain score of the hip between the two groups $(P>0.05)$.

\section{Conclusions}

Two-stage THA using antibiotic-loaded spacer implantation is an effective method for the treatment of advanced SA of the hip in adults due to it can control infection and significantly improve functional hip.

\section{Background}

Septic arthritis (SA) is a devastating disease in adults with high morbidity and mortality [1], which mainly affect the knee and hip joints [2]. Patients with ineffective treatment may lead to osteonecrosis of the femoral head, osteomyelitis, systemic sepsis, leg-length discrepancy, and later osteoarthritis of the hip joint [3]. Despite the surgical treatment, the occurrence of poor outcomes can still reach to $22 \%$ [4]. Primary SA of the hip is defined as infection of the hip joint that developed in the absence of a fracture, insertion of an implant or prosthesis, and surgical procedure. Until now, multiple treatment options are available, such as repeated aspirations, open arthrotomy, arthroscopic drainage and two-stage total hip arthroplasty (THA) [5, 6]. However, treatment for advanced SA of the hip can be confronted with great challenges. 
When SA with bone involvement, more aggressive surgery have been conducted. For example, a previous study by Girdlestone et al. [7] has reported that resection arthroplasty is effective treatment for SA of the hip. However, it may be associated with poor function outcomes. Subsequent report has found that the use of antibiotic-loaded spacers in two-stage THA may be considered as a suitable method for the treatment of primary SA in adults [8]. In addition, Li et al. [9] have reported that two-stage THA using debridement or femoral head resection with antibiotic-loaded spacer implantation is effective in the treatment of advanced active tuberculosis of the hip and have demonstrated that the outcomes of two procedures are consistent. Therefore, it is necessary for us to further demonstrate the treatment of between debridement and antibiotic-loaded spacer implantation in two-stage of THA in adults.

The aim of this study was to compare the outcomes of hip using debridement and femoral head resection with antibiotic-loaded spacer implantation in advanced SA of the hip in adults. Our study demonstrated that two-stage THA using antibiotic-loaded spacer implantation is an effective method for the treatment of advanced septic arthritis of the hip in adults. These findings of this study may provide a new treatment for primary advanced SA of hips in adults.

\section{Methods}

\section{Patients}

We conducted a single-center, retrospective cohort study of patients with follow-up. From January 2014 to December 2017, 21 patients with SA of the hip were eligible for enrolment in our study. Among them, 1 patient decided to leave the hospital and was treated in the local hospital after being diagnosed. A total of 20 patients were finally included. Inclusion criteria were patients who 1) were aged $\geq 18$ years; 2) had a definite diagnosis of acute SA of the hip joint; 3) were advanced SA with destruction of hip joint; and 4) had a set of complete medical records (including nursing records), preoperative and postoperative radiographs. Exclusion criteria were patients who 1) were early SA of hip; 2) were multiple arthritis; 3) had incomplete medical records; 4) were not willing to be followed up, 5) had immune deficiency.

\section{Patient diagnosis}

Patients with SA of the hip had clinical symptoms of infection including pain, sudden chills and fever; local swelling, and limited range of motion. In addition, complete blood cell count (CBC), C-reactive protein (CRP) and erythrocyte sedimentation rate (ESR) were measured. X-ray, CT and MRI were also performed. X-ray showed narrowing of the hip joint space for advanced SA of the hip. CT could detect local edema, osteonecrosis, osteomyelitis, and hip bone destruction. MRI provided good resolution for the detection of joint effusion, bone differentiation, and soft tissue infection. MRI findings of SA patients include joint effusion, cartilage and bone destruction, soft tissue abscess and bone edema [10] (Fig. 13). The diagnosis of SA synthesized the results of these tests. If the patient was suspected of having septic arthritis, PPD test, Brucella agglutination test and needle aspiration of the hip were then added. The 
patient should accept an procedure according to patent's willing once sepsis is confirmed via aspiration [11].

\section{Surgical technique}

All of the 20 patients (20 hips) underwent two-stage surgeries. Among them, 9 cases underwent debridement, 11 cases underwent antibiotic-loaded spacer implantation in the first stage. After a period of time, THA was conducted in all patients in the second stage. Therefore, two procedures were named as debridement with THA group and spacer implantation with THA group, respectively. Patients chose one of two procedures according to patent's willing. All operations were performed by four professional surgeons in our department.

During the first stage, posterolateral approach was performed in all patients. Debridement was performed in 9 patients (9 hips) and femoral head resection with antibiotic-loaded spacer implantation were performed in 11 patients (11 hips). The inflamed soft tissues, necrotic bones, abscesses, and sinus tracts were totally debrided during the first stage. Then the specimens were taken for pathological examination and tissue bacterial culture. For patients undergoing debridement, irrigation tube were placed in operation and continued to be rinsed with gentamicin solution after operation but not in patients with antibioticloaded spacer implantation. For patients undergoing femoral head resection and antibiotic-loaded spacer implantation, femoral head were removed and a cement spacer containing gentamicin were installed (Fig. 1). Susceptible antibiotic drugs were given to patients based on the results of bacterial culture until serological results return to normal. Symptoms disappeared 2 weeks later. Empirical antibiotics (ceftriaxone) was used in patients with negative bacterial culture results $[11,10]$. CBC, CRP and ESR were detected for weekly regular monitoring. Patients stopped antibiotic usage and discharged once the vital signs and serological indicators was basically normal and the infection was eradicated. If there was pain in the affected limb within 24-48 hours after operation, patient was conducted for traction of the affected limb. After 48 hours, patient strengthened functional exercise [10, 12].

During the second-stage, all procedures were performed via the posterolateral approach, with the patient in a lateral position. When the joint was opened, no pus and no inflammatory edema of soft tissue around the joint was confirmed under the naked eye. Then the hypertrophic soft tissue and scar was removed as thoroughly as possible in the direct visualization. Odophor and hydrogen peroxide were used ito immerse the hip joint cavity to reduce the probability of infection. Titanium Acetabular Reconstruction Cup was used for 2 cases with deficient acetabular wall, which was fixed with screws. Then acetabular impactors were used to perform impaction graft. The gap between cup and host bone was completely filled by allogeneic morselized bone. On completion of cage placement and bone grafts, an acetabular polyethylene liner was fixed with high radiopaque bone cement conting gentamicin. In addition, central acetabular defect in 3 patients were performed graft impaction using acetabular impactors, and a larger acetabular cup was implanted. Besides, 1 patient had a high hip dislocation secondary to SA of the hip. Structural autograft obtained from the femoral neck was implanted on the patien's upper acetabular bone and fixed with screws. The remaining 15 patients were operated normal THA. The usage of antibiotics 
dependeded on the susceptibility of the first-stage organism identified in joint fluid culture. Antibiotics were chosen empirically if no organism was identified.

\section{Data collection}

The scanning edition of electronic medical records were reviewed in detail to retrieve pertinent information, including demographic data (gender, age, height and weight), the preoperative and postoperative clinical evaluation (CBC, CRP and ESR), imaging evaluation (X-ray, CT and MRI), Harris hip score and VAS pain score. Clinical data were all recorded during follow-up.

\section{Follow up}

Anteroposterior and lateral radiographs of the hip, full-length view of the lower extremities, CT and MRI of the hip were taken for the pre-operation. In our department, patients were required to have regular followup in the 1st month, the 3rd month, the 6th month and the 12th month after operation. Anteroposterior and lateral radiographs of the hip were taken at each follow-up. All of the 20 patients had adequate preoperative and postoperative imaging examinations.

\section{Statistical analysis}

All data were performed using SPSS 19.0 software (IBM, Armonk, NY, USA). Quantitative data were described using means \pm standard deviation (SD). Mann-Whitney $U$ test was used to compare continuous categorical variables, including Harris hip score and VAS pain score between the two groups. A P value of less than 0.05 were considered statistically significant.

\section{Results}

All of 20 patients had postoperative follow-up. In our study, 20 hips (9 hips for debridement with THA procedure; 11 hips for antibiotic-loaded spacer implantation with THA procedure) of 20 patients (16 males; 4 females) were analyzed. The mean age was $50.60 \pm 10.82$ years. There were no significant differences in laboratory indexes, Harris hip scores, VAS pain scores and Body Mass Index (BMI) between the two groups preoperatively (Table 1 ). 
Table 1

Baseline characteristics in two groups

\begin{tabular}{|lllc|}
\hline & $\begin{array}{l}\text { Debridement } \\
\text { with THA group }(\mathbf{n}=9)\end{array}$ & $\begin{array}{l}\text { Spacer implantation } \\
\text { with THA group }(\mathbf{n}=11)\end{array}$ & P value \\
\hline Age(years) & $47.89 \pm 10.34$ & $52.82 \pm 11.17$ & .270 \\
\hline CRP $(\mathrm{mg} / \mathrm{L})$ & $48.44 \pm 45.58$ & $67.41 \pm 69.39$ & .569 \\
\hline ESR $(\mathrm{mm} / \mathrm{h})$ & $68.67 \pm 31.23$ & $56.36 \pm 23.56$ & .323 \\
\hline $\begin{array}{l}\text { Leucocyte count } \\
\left(10^{\wedge} 9 / \mathrm{L}\right)\end{array}$ & $8.30 \pm 2.06$ & $7.26 \pm 1.53$ & .271 \\
\hline Neutrophil count $(\%)$ & $67.68 \pm 5.10$ & $64.30 \pm 12.20$ & .621 \\
\hline BMI $\left(\mathrm{kg} / \mathrm{m}^{2}\right)$ & $23.05 \pm 3.49$ & $23.57 \pm 3.13$ & .595 \\
\hline Harris hip score & $29.56 \pm 4.00$ & $28.45 \pm 3.86$ & .541 \\
\hline VAS pain score & $4.67 \pm 0.71$ & $5.36 \pm 1.12$ & .151 \\
\hline $\begin{array}{l}\text { Time of symptoms pre- } \\
\text { operation(day) }\end{array}$ & $44.22 \pm 24.45$ & $99.55 \pm 81.53$ & \\
\hline
\end{tabular}

In the first stage, the results of bacterial culture of joint fluid in 20 patients were Staphylococcus aureus (4 cases), Brucella (1 case), Mycobacterium tuberculosis (1 case), Klebsiella pneumoniae (1 case) and the rest were negative. In the first stage of surgery, the two groups had similar operation duration and blood loss. BMI increased before the second stage of operation compared with that before the first stage of operation, indicating that the patients recovered using after operation in both groups (Table 2). The mean follow-up time was $28.22 \pm 14.80$ months in the debridement with THA group and $29.09 \pm 10.80$ months in the spacer implantation with THA group, respectively. No recurrence of SA occurred after the secondstage of two-stage THA. No loosening or dislocation of prosthesis, thrombosis or death were found in all patients during the follow-up period. The symptoms of the patients were greatly improved. The Harris hip score increased in two groups after two-stage THA surgery $(28.45 \pm 3.86,29.56 \pm 4.00$ vs $94.36 \pm 2.34$, $90.33 \pm 4.85)$, and the VAS pain score decreased after two-stage THA surgery $(5.36 \pm 1.12,4.67 \pm 0.71$ vs $0.09 \pm 0.30,0.44 \pm 0.53)$.

We also observed many differences between the two methods in Table 2. For the first stage, the mean duration of postoperative hospitalization in the debridement with THA group (19.00 \pm 4.95 days) was significantly longer than that in the spacer implantation with THA group $(12.09 \pm 2.17$ days $)(P=0.001)$. The patients with antibiotic-loaded spacer implantation had a better recovery of hip joint function. However, the patient with debridement has more complications including traumatic arthritis (Fig. 2), high dislocation of hip joint (Fig. 4) and then needed THA. The mean Harris hip score in the debridement with THA group was lower than in the spacer implantation with THA group after first stage of two-stage THA (all $\mathrm{P}<0.05$ ). The duration between the two stages of operation in the debridement with THA group 
(10.30 \pm 8.70 months) was longer than that in the spacer implantation with THA group $(3.43 \pm 1.50$ months) $(P=0.039)$. Before the THA surgery, the mean leg-length discrepancy was $2.80 \pm 2.03 \mathrm{~cm}$ in debridement with THA and $0.50 \pm 0.23 \mathrm{~cm}$ in spacer implantation with THA $(P<0.05)$. In the second stage, the operation time of the debridement with THA group $(175.00 \pm 68.74 \mathrm{~min})$ was significantly longer than that of the spacer implantation with THA group $(131.36 \pm 30.67 \mathrm{~min})(P=0.047)$.

For the latest follow-up, Harris hip score was $90.33 \pm 4.85$ points in the debridement with THA group and $94.36 \pm 2.34$ points in the spacer implantation with THA group $(P=0.027)$. There were no significant differences in the mean VAS score between the debridement with THA group $(0.44 \pm 0.53)$ and antibioticloaded spacer implantation with THA group $(0.09 \pm 0.30)(P=0.077)$, but 4 of the 9 patients $(44.44 \%)$ who received the debridement with THA procedure had occasional hip discomfort or mild aches, compared with 1 of the 11 patients (9.10\%) who received the spacer implantation with THA procedure (Fig. 3) (Table 2) . 
Table 2

Comparison of perioperative outcomes in two groups

\begin{tabular}{|c|c|c|c|}
\hline & $\begin{array}{l}\text { Debridement } \\
\text { with THA group }(n=9)\end{array}$ & $\begin{array}{l}\text { Spacer implantation } \\
\text { with THA group }(n=11)\end{array}$ & $P$ value \\
\hline PODFSH (day) & $19.00 \pm 4.95$ & $12.09 \pm 2.17$ & $.001^{\star}$ \\
\hline IBTSOS (day) & $10.30 \pm 8.70$ & $3.43 \pm 1.50$ & $.039 *$ \\
\hline \multicolumn{4}{|l|}{$\mathrm{BMI}\left(\mathrm{kg} / \mathrm{m}^{2}\right)$} \\
\hline First-stage & $23.05 \pm 3.49$ & $23.57 \pm 3.13$ & .595 \\
\hline Second-stage & $25.39 \pm 4.46$ & $25.13 \pm 2.87$ & .569 \\
\hline \multicolumn{4}{|l|}{ Duration of operation (min) } \\
\hline First-stage operation & $137.22 \pm 39.06$ & $130 \pm 31.06$ & .879 \\
\hline Second-stage operation & $175.00 \pm 68.74$ & $131.36 \pm 30.67$ & $.047 *$ \\
\hline \multicolumn{4}{|l|}{ Blood loss (ml) } \\
\hline First-stage operation & $438.89 \pm 119.32$ & $595.45 \pm 322.84$ & .591 \\
\hline Second-stage operation & $1211.11 \pm 1842.85$ & $754.55 \pm 697.66$ & .697 \\
\hline \multicolumn{4}{|l|}{ Harris hip score } \\
\hline First-stage preoperative & $29.56 \pm 4.00$ & $28.45 \pm 3.86$ & .541 \\
\hline First-stage out of hospital & $61.67 \pm 3.04$ & $73.55 \pm 4.16$ & $.000 *$ \\
\hline Second-stage preoperative & $74.44 \pm 3.40$ & $80.64 \pm 2.66$ & $.001 *$ \\
\hline Second-stage out of hospital & $81.78 \pm 2.22$ & $84.18 \pm 2.14$ & $.049 *$ \\
\hline Latest follow-up & $90.33 \pm 4.85$ & $94.36 \pm 2.34$ & $.027 *$ \\
\hline \multicolumn{4}{|l|}{ VAS pain score } \\
\hline First-stage preoperative & $4.67 \pm 0.71$ & $5.36 \pm 1.12$ & .151 \\
\hline First-stage out of hospital & $1.44 \pm 0.53$ & $1.36 \pm 0.51$ & .721 \\
\hline Second-stage preoperative & $1.67 \pm 0.71$ & $1.18 \pm 0.41$ & .077 \\
\hline Latest follow-up & $0.44 \pm 0.53$ & $0.09 \pm 0.30$ & .077 \\
\hline Length discrepancy & $2.80 \pm 2.03$ & $0.50 \pm 0.23$ & $.000 \star$ \\
\hline Follow-up (month) & $28.22 \pm 14.80$ & $29.09 \pm 10.80$ & .518 \\
\hline
\end{tabular}




\section{Discussion}

Primary SA of the hip is rare in adults [13], which lead to serious complications and significant morbidity and mortality if it is not diagnosed and treated promptly [14]. Two-step total hip arthroplasty has been used when the hip suffer from severe destruction [15]. In our study, we found that a main type of microorganism is staphylococcus aureus. Furthermore, we compared the effect of between debridement and femoral head resection with anti-loaded spacer implantation in the first stage of surgery. The finding showed that Two-stage of THA using anti-loaded spacer implantation was superior to Two-stage of THA using debridement with regard to mean leg-length discrepancy of patients and Harris hip scores.

The most common causative microorganism related to SA of hip is Staphylococcus aureus and Streptococcus pyogenes $[16,17]$. In our cases which was diagnosed with a specific bacterial infection, Staphylococcus aureus accounted for $57.14 \%$. In the joint with pus, the positive rate of bacterial culture in synovial fluid is about $80 \%[18,1]$. However in our study, the positive rate of bacterial culture of synovium fluid was $25 \%$. The possibility for low culture positive rate was that patients had been given antibiotics orally or intravenously before admission. The reason for using antibiosis might be used to control joint infection as well as prevent further septic metastases [19].

Generally, physicians typically have 2 tasks consisted of infection control and hip function restore when treating SA of hip. Therefore, surgery and antibiotics are key factors for treating for primary SA of the hip. Previous studies have demonstrated that Two-stage THA is considered as a suitable method for the treatment of primary SA. For example, Bauer et al. [20] have concluded that two-stage THA had a good treatment for the infection in the treatment of evolutive SA. A similar study has also demonstrated that two-stage THA using interval spacer can effective control the rate of infection [21]. In our study, both menthods of Two-stage THA using debridement or antibiotic-loaded spacer implantation achieved the goal of eliminating infection. In addition, we observed that the second stage was less difficult and pateints had less complications when using antibiotic-loaded spacer in the first stage of hip arthroplasty. However, the patients had more complications including traumatic arthritis and high dislocation of hip joint when using debridement in the first stage of hip arthroplasty. Those data suggested that two-stage THA using antibiotic-loaded spacer implantation had less complications in treating for advanced primary SA of the hip.

Subsequent study has revealed that two-stage THA using interval spacer can improve the Harris Hip Score [22]. In addition, Yoon et al. [23] have reported that two-stage THA could reduce the mean leg length discrepancy from 2.95 to $0.8 \mathrm{~cm}$. Furthermore, two-stage THA using debridement or anti-loaded spacer implantation can treat effectively for patients with advanced active tuberculosis of the hip and there were no significant differences between the two procedures [9]. In our study, patients who underwent debridement had longer hospital stay than patients underwent who anti-loaded spacer implantation after the first stage of surgery. In the second stage of THA, patients in the debridement group had longer operation duration and more blood loss. The recovery of patients in the debridement group was slower than that in the anti-loaded spacer implantation group. In addition, pateints who received Two-stage THA 
using anti-loaded spacer implantation had higher Harris hip score, smaller mean leg-length discrepancy. During follow-up, several patients who received Two-stage THA using debridement occasionally felt slight pain in the front of the hip or thigh at the latest follow-up, however the VAS pain score of the hip had no significant difference. Those abovementioned findings suggested Two-stage THA using anti-loaded spacer implantation had good effect on function of the hip.

There were several limitations in this study. For example, This study was retrospective and conducted in a single center medical institution with a small sample. Those abovementioned situation may result in sampling error and conclusions may be influence by unrelated factors. Therefore, large randomized controlled study should be carried out in the future.

\section{Conclusion}

The present study suggested that for adult patients with advanced SA of the hip, the procedure of twostage THA using anti-loaded spacer implantation can effectively control infection and significantly improve functional hip. Therefore, Two-stage THA using anti-loaded spacer implantation may be a promising option for the treatment of advanced primary SA of the hip in adults.

\section{List Of Abbreviations}

SA, septic arthritis; THA, total hip arthroplasty; VAS, visual analogue scale; CBC, complete blood cell count; CRP, C-reactive protein; ESR, erythrocyte sedimentation rate.

\section{Declarations}

\section{Ethical Approval and Consent to participate}

The study was approved by the Institutional Ethics Committee of the Third Hospital of Hebei Medical University (2020-004-1) and allowed exemption from informed consent. All procedures were performed in accordance with the World Medical Association's Declaration of Helsinki.

\section{Consent for publication}

All of participants were given written informed consent.

\section{Availability of Data and Materials}

All data generated or analysed during this study are included in this published article.

\section{Competing Interest}

The authors indicated no potential conflicts of interest. 
Not applicable.

\section{Authors' contributions}

ZZL and HJL conceived of the study, participated in data collection, analysis, and interpretation of the results and writing of the manuscript. CCW, XKL and MXY contributed to data collection, and interpretation of the results. All authors read and approved the final manuscript and consented to publish this manuscript.

\section{Acknowledgements}

Not applicable.

\section{References}

1. Margaretten ME, Kohlwes J, Moore D, Bent S. Does this adult patient have septic arthritis? Jama 2007;297:1478-88.

2. George J, Chandy VJ, Premnath J, Hariharan TD, Oommen AT, Balaji V, et al. Microbiological profile of septic arthritis in adults: Lessons learnt and treatment strategies. Indian J Med Microbiol 2019;37:2933.

3. Fabry G, Meire E. Septic arthritis of the hip in children: poor results after late and inadequate treatment. J Pediatr Orthop 1983;3:461-6.

4. Kao FC, Hsu YC, Liu PH, Tu YK, Jou IM. High 2-year mortality and recurrent infection rates after surgical treatment for primary septic arthritis of the hip in adult patients: An observational study. Medicine (Baltimore) 2019;98:e16765.

5. Xu G, Spoerri M, Rutz E. Surgical treatment options for septic arthritis of the hip in children. Afr J Paediatr Surg 2016;13:1-5.

6. Luo Y, Yang Z, Yeersheng R, Li D, Kang P. Clinical outcomes and quality of life after total hip arthroplasty in adult patients with a history of infection of the hip in childhood: a mid-term follow-up study. 2019;14:38.

7. Girdlestone GR. Acute pyogenic arthritis of the hip: an operation giving free access and effective drainage. 1943. Clin Orthop Relat Res 2008;466:258-63.

8. Morgan DS, Fisher D, Merianos A, Currie BJ. An 18 year clinical review of septic arthritis from tropical Australia. Epidemiol Infect 1996;117:423-8.

9. Li L, Chou K, Deng J, Shen F, He Z, Gao S, et al. Two-stage total hip arthroplasty for patients with advanced active tuberculosis of the hip. J Orthop Surg Res 2016;11:38.

10. García-Arias M, Balsa A, Mola EM. Septic arthritis. Best practice \& research Clinical rheumatology 2011;25:407-21.

11. Mathews CJ, Weston VC, Jones A, Field M, Coakley G. Bacterial septic arthritis in adults. Lancet 2010;375:846-55. 
12. Kirchhoff C, Braunstein V, Paul J, Imhoff AB, Hinterwimmer S. Septic arthritis as a severe complication of elective arthroscopy:clinical management strategies. Patient Saf Surg 2009;3:6.

13. Schroder JH, Kruger D. Arthroscopic Treatment for Primary Septic Arthritis of the Hip in Adults. 2016;2016:8713037.

14. KELLY PJ, MARTIN WJ, COVENTRY MB. Bacterial (suppurative) arthritis in the adult. JBJS 1970;52:1595-602.

15. Chen CE, Wang JW, Juhn RJ. Total hip arthroplasty for primary septic arthritis of the hip in adults. Int Orthop 2008;32:573-80.

16. Geipel U. Pathogenic organisms in hip joint infections. Int J Med Sci 2009;6:234-40.

17. Goldenberg DL. Septic arthritis. The lancet 1998;351:197-202.

18. Long B, Koyfman A, Gottlieb M. Evaluation and Management of Septic Arthritis and its Mimics in the Emergency Department. Western Journal of Emergency Medicine 2019;20:331.

19. Anagnostakos K, Duchow L, Koch K. Two-stage protocol and spacer implantation in the treatment of destructive septic arthritis of the hip joint. Archives of orthopaedic and trauma surgery 2016;136:899906.

20. Bauer T, Lacoste S, Lhotellier L, Mamoudy P, Lortat-Jacob A, Hardy P. Arthroplasty following a septic arthritis history: a 53 cases series. Orthop Traumatol Surg Res 2010;96:840-3.

21. Anagnostakos K, Duchow L, Koch K. Two-stage protocol and spacer implantation in the treatment of destructive septic arthritis of the hip joint. Arch Orthop Trauma Surg 2016;136:899-906.

22. Shen $H$, Wang QJ, Zhang XL, Jiang Y. Novel articulating medullary-sparing spacer for the treatment of infectious hip arthritis. Orthopedics 2013;36:e404-8.

23. Yoon PW, Kim JI, Kim DO, Yu CH, Yoo JJ, Kim HJ, et al. Cementless total hip arthroplasty for patients with Crowe type III or IV developmental dysplasia of the hip: two-stage total hip arthroplasty following skeletal traction after soft tissue release for irreducible hips. Clin Orthop Surg 2013;5:16773.

\section{Figures}



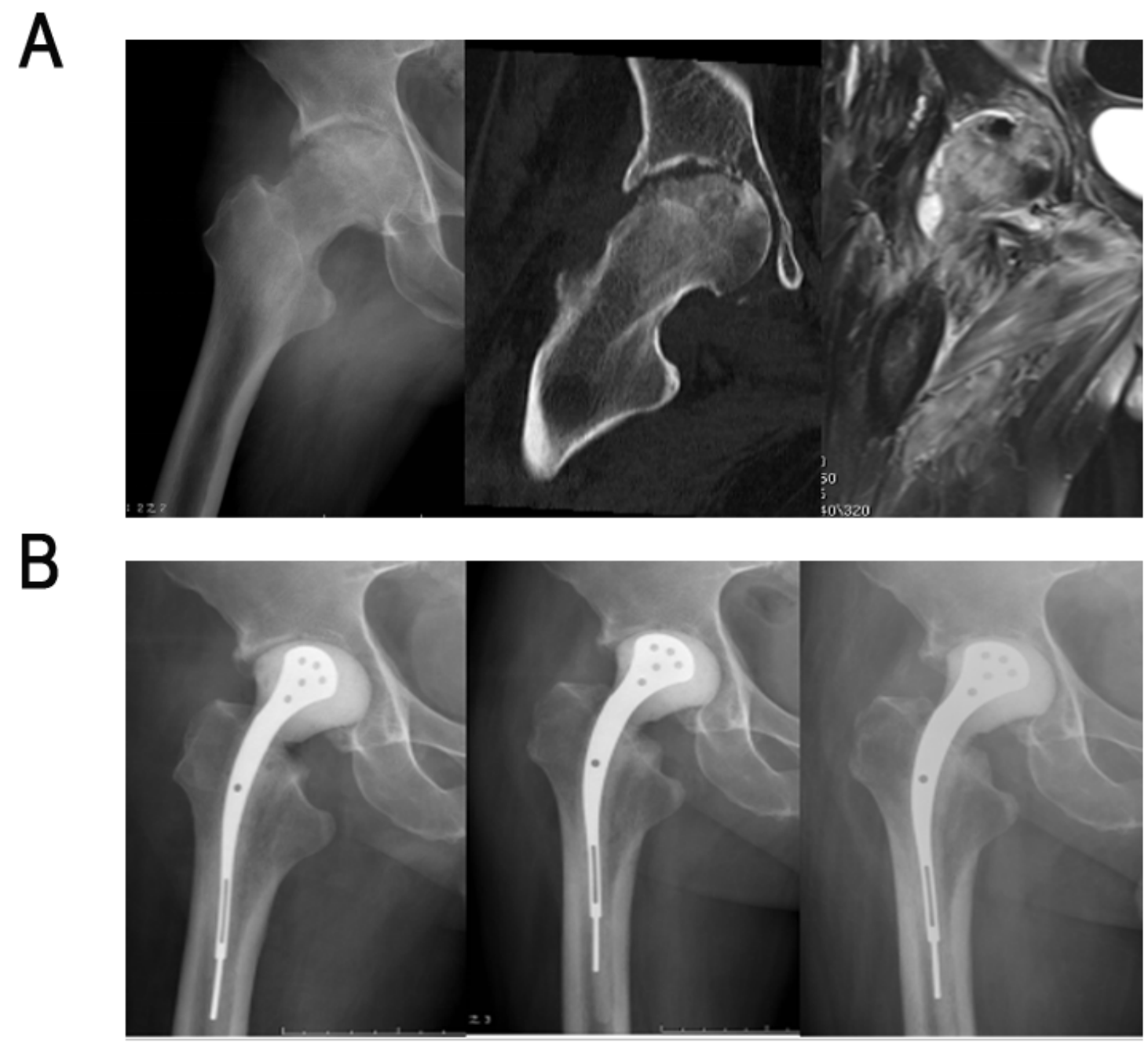

C

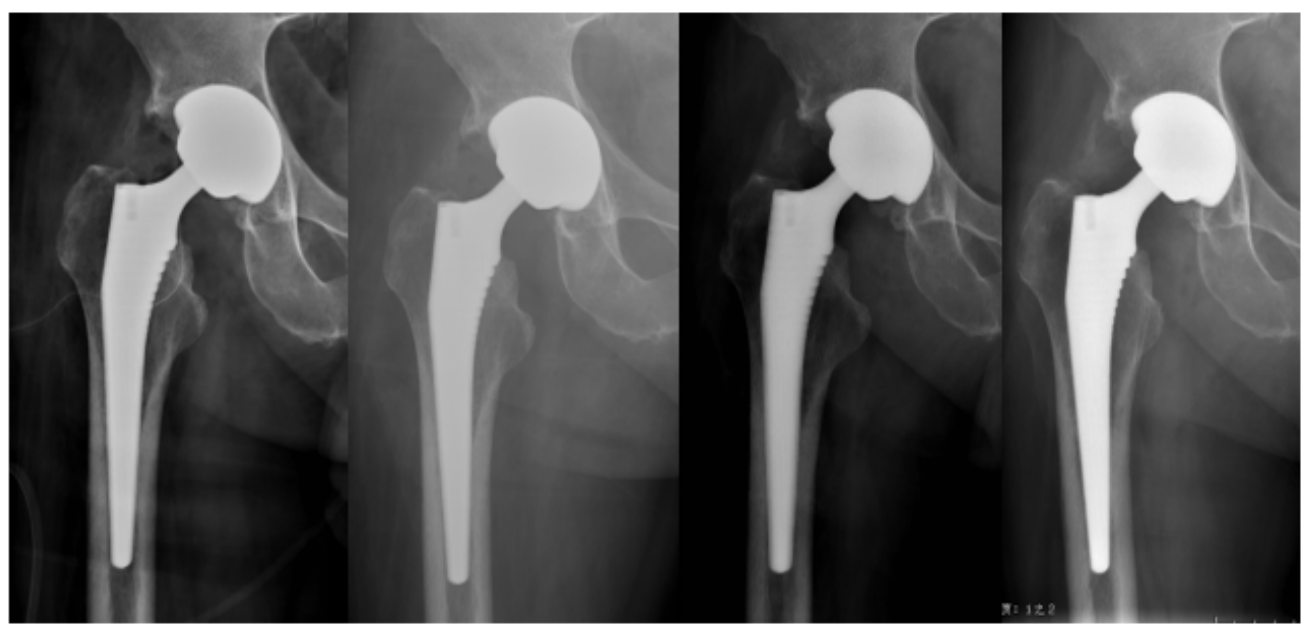

\section{Figure 1}

Radiographs of a 49-year-old man with pain in his right hip. (A) Anteroposterior X-ray and coronal CT images of pelvis showed narrowing of hip joint space and destruction of bone. coronal MRI (cut) of the pelvis showed diffuse edema of the right hip joint involving almost all muscles of the right hip. (B) The femoral head was excised and implanted with an antibiotic cement prosthesis: 5 days, 1 month and 3 
months after operation respectively. (C) The postoperative imaging findings of hip replacement at 0 day, 1 month, 3 months and 19 months, respectively.
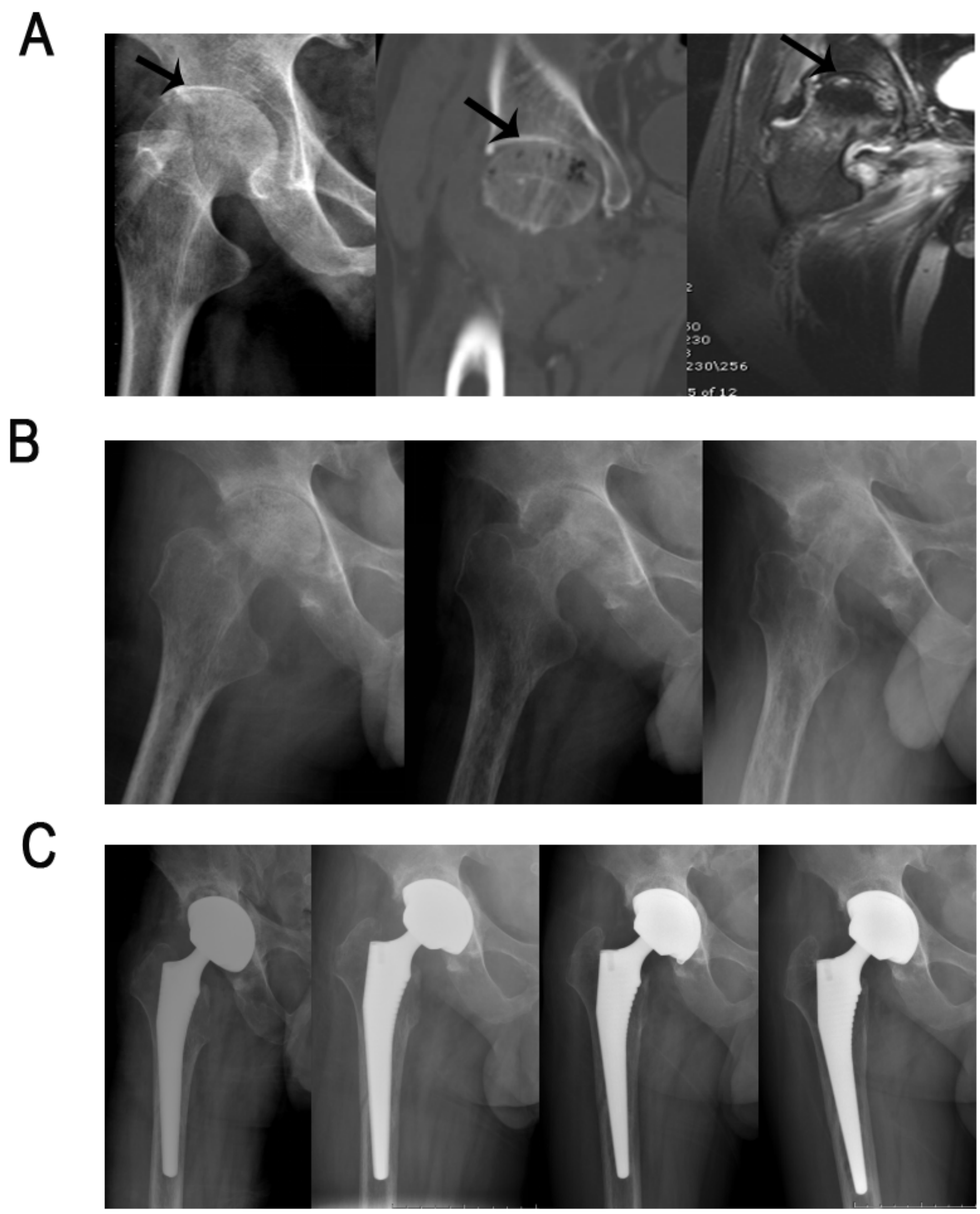

\section{Figure 2}

Radiographs of a 43-year-old man with pain in his right hip joint. (A) Anteroposterior X-ray and coronal CT images of pelvis showed that the space of hip joint became narrow and articular cartilage disappeared (arrow). coronal MRI of the pelvis showed diffuse edema of the right femoral head. (B) X-ray examination 
of hip joint at 4 months, 12 months and 15 months after debridement operation. (C) The postoperative images of hip replacement at 7 days, 2 months, 5 months and 15 months, respectively.

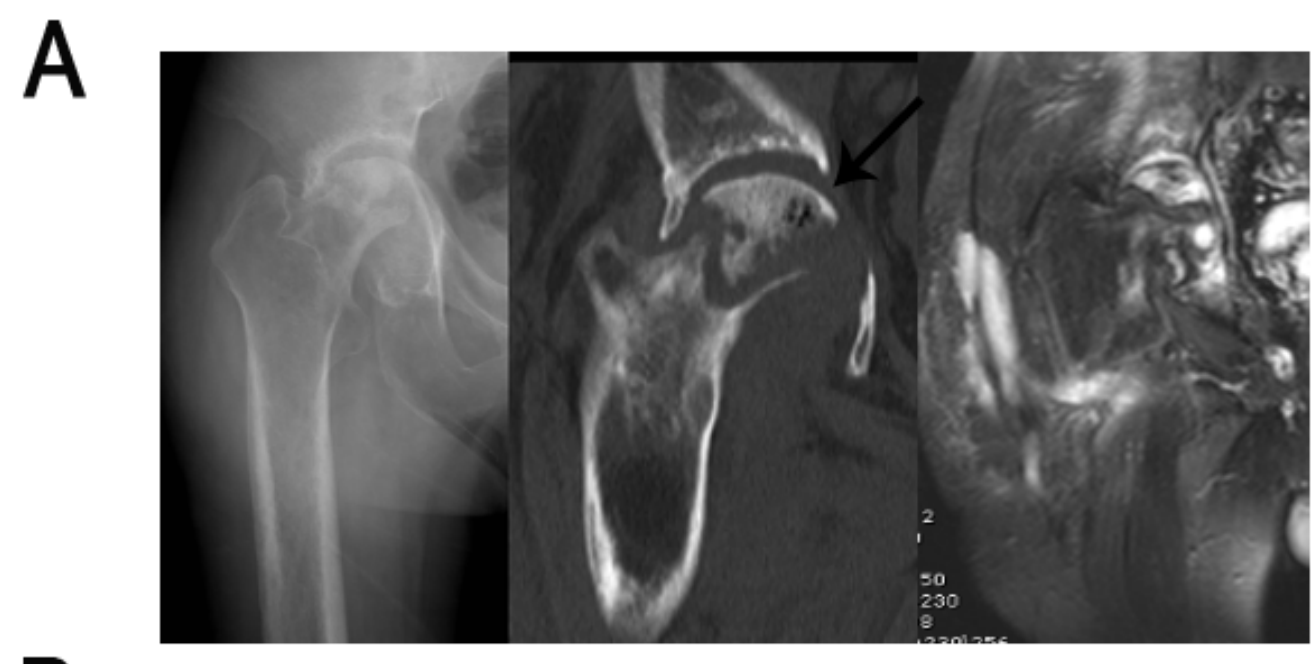

B
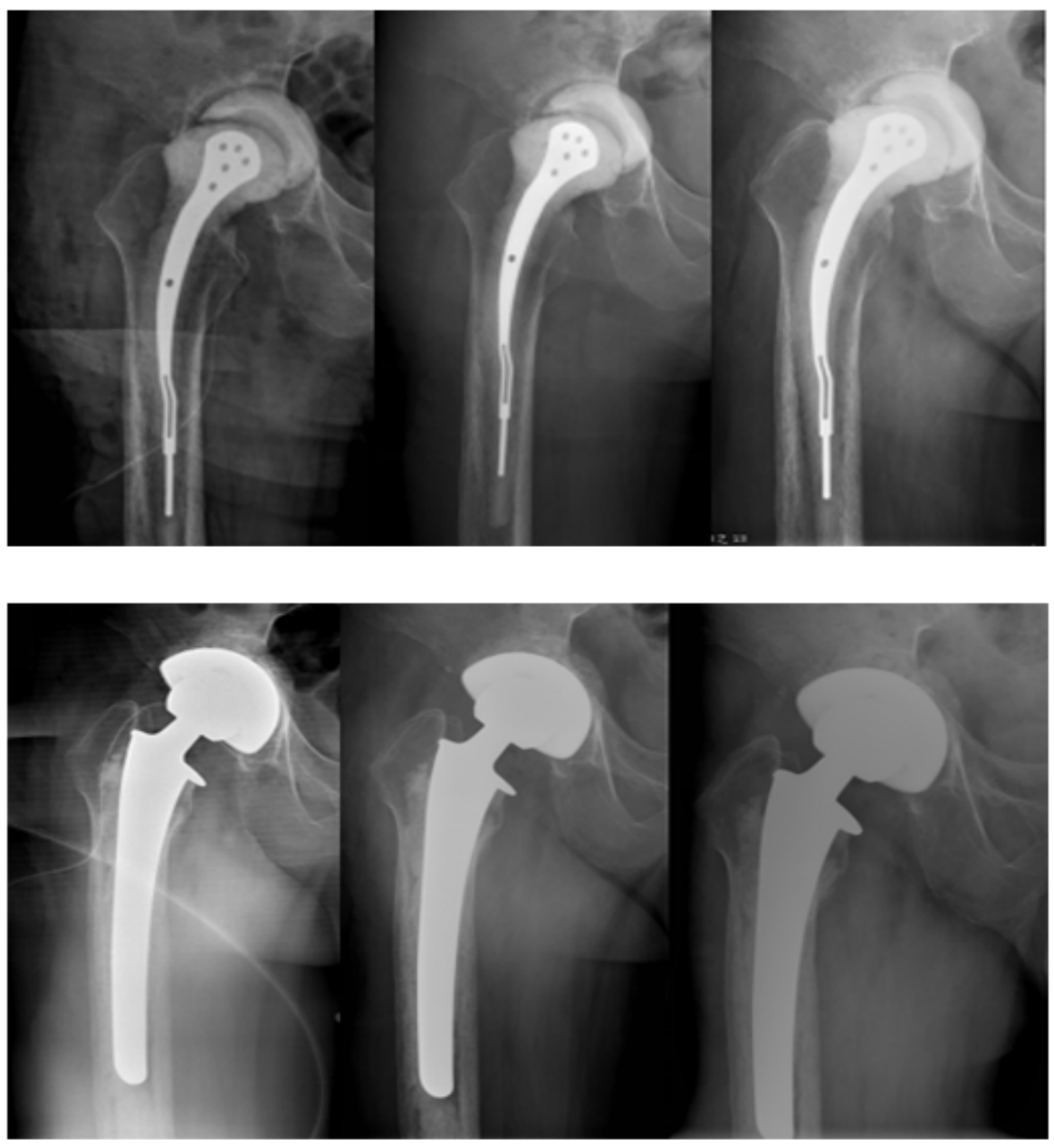

Figure 3

Radiographs of a 54-year-old man with right hip joint pain and severe limitation of movement. (A) Anteroposterior X-ray and coronal CT images of pelvis showed that the right acetabulum was invaginated and the acetabulum wall was damaged (arrow). Coronal MRI of the pelvis showed inflammatory changes 
in the right hip joint. (B) The postoperative imaging of femoral head resection with antibiotic cement implantation at the same day, 1 month and 6 months, respectively. (C) The imaging findings after THA at 0 day, 1 month and 12 months, respectively.

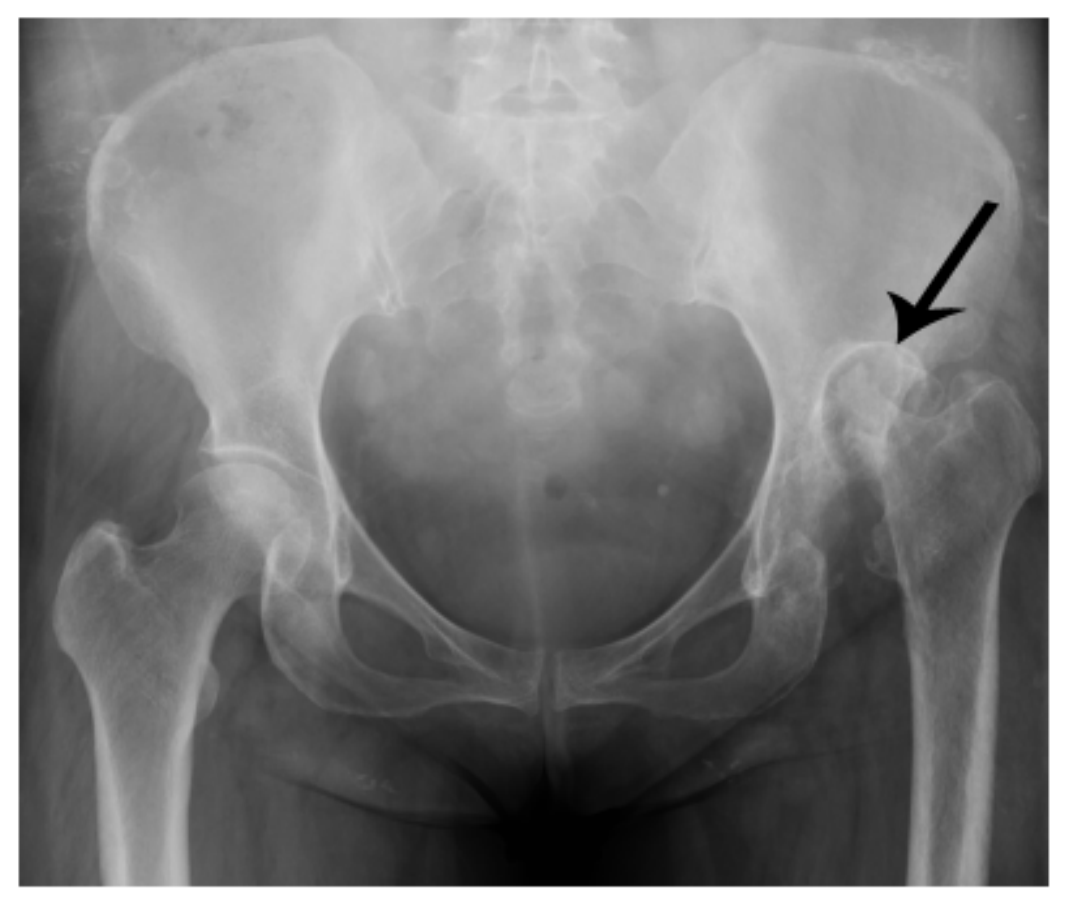

Figure 4

Anteroposterior X-ray images of the pelvis showed that the left femoral head had been absorbed, and the rest of the femoral neck had been dislocated and formed a pseudarthrosis (arrow). 